\title{
A HEAVY-TRAFFIC THEOREM FOR THE GI/G/1 QUEUE WITH A PARETO-TYPE SERVICE TIME DISTRIBUTION
}

\author{
J.W. COHEN ${ }^{1}$ \\ $C W I$ \\ P.O. Box 94079 \\ 1090 GB Amsterdam, The Netherlands
}

(Received July, 1997; Revised November, 1997)

For the GI/G/1 queueing model with traffic load $a<1$, service time distribution $B(t)$ and interarrival time distribution $A(t)$, whenever for $t \rightarrow \infty$

$$
1-B(t) \sim \frac{c}{(t / \beta)^{\nu}}+\mathrm{O}\left(e^{-\delta t}\right), c>0,1<\nu<2, \delta>0,
$$

and

$$
\int_{0}^{\infty} t^{\mu} d A(t)<\infty \text { for } \mu>\nu,
$$

$(1-a)^{\frac{1}{\nu-1}} \mathbf{w}$ converges in distribution for $a \uparrow 1$. Here $\mathbf{w}$ is distributed as the stationary waiting time distribution. The L.-S. transform of the limiting distribution is derived and an asymptotic series for its tail probabilities is obtained. The theorem actually proved in the text concerns a slightly more general asymptotic behavior of $1-B(t), t \rightarrow \infty$, than mentioned above.

Key words: GI/G/1 Model, Pareto-Type Service Time Distribution, Heavy-Traffic Theorem, Tail Asymptotics.

AMS subject classifications: $90 \mathrm{~B} 22,60 \mathrm{~K} 25$.

\section{Introduction}

For the GI/G/1 queue, denote by $A(t)$ and $B(t)$ the interarrival time distribution and service time distribution, respectively, and by $a$ the traffic load, with $a<1$.

The distribution $B(t)$ is said to have a Pareto-type tail if: for $t \rightarrow \infty$,

\footnotetext{
${ }^{1}$ Work carried out under project LRD. 


$$
\begin{gathered}
1-B(t)=\frac{c}{(t / \beta)^{\nu}}+\sum_{n=1}^{N} \frac{c_{n}}{(t / \beta)^{\nu}}+\mathrm{O}\left(e^{-\delta t}\right), \\
\left.1<\nu<2, \quad \beta:=\int_{0}^{\infty} t d B(t)\right), \quad c>0, \quad \delta>0, \\
c_{n} \geq 0, \quad \nu_{n}>\nu, \quad N \text { a finite integer } \geq 1 .
\end{gathered}
$$

w shall denote a stochastic variable with distribution $W(t)$, the stationary distribution of the actual waiting time of the GI/G/1 model.

Write

$$
\Delta:=\left[\frac{1-a}{a} \frac{\Gamma(\nu) \sin (v-1) \pi}{c \pi}\right]^{\frac{1}{\nu-1}}
$$

here $\Gamma(\cdot)$ is the gamma function and $x^{\alpha}, \alpha$ real, is defined by its principal value, i.e., it is positive for $x$ positive.

Theorem: When $B(t)$ has a Pareto-type tail as specified in (1.1) and when

$$
\int_{0}^{\infty} t^{\mu} d A(t)<\infty \text { for } a \mu>\nu
$$

then the stochastic variable $(1-a)^{\frac{1}{\nu-1}} \mathbf{w} / \beta$ converges for a 1 in distribution, and

$$
\lim _{a \uparrow 1} \mathrm{E}\left\{\mathrm{e}^{-\rho \Delta \mathbf{w} / \beta}\right\}=\frac{1}{1+\rho^{\nu-1}}, \quad \operatorname{Re} \rho \geq 0 ;
$$

the right-hand side of (1.4) is the Laplace-Stieltjes transform of a true probability distribution $R_{\nu-1}(t)$ with support $(0, \infty)$; and for $t \rightarrow \infty$ and every finite $H \in\{1,2, \ldots\}$,

$1-R_{\nu-1}(t)=\frac{1}{\pi} \sum_{n=1}^{H}(-1)^{n-1} \frac{\Gamma(n(\nu-1)) \sin n(\nu-1) \pi}{t^{n(t-1)}}+\mathrm{O}\left\{t^{-(H+1)(\nu-1)}\right\}$.

For a special class of Pareto-type tailed service time distributions, the theorem has been derived for the M/G/1 model in [2]. The distribution $R_{\nu-1}(t)$ is called the Kovalenko distribution, cf. [9]. For $\nu=1 \frac{1}{2}$ we have, cf. [1],

$$
R_{1 / 2}(t)=1-\frac{2}{\sqrt{\pi}} \mathrm{e}^{t} \operatorname{Erfc}\left(t^{1 / 2}\right), \quad t>0
$$

with

$$
\operatorname{Erfc}(x)=\int_{x}^{\infty} \mathrm{e}^{-u^{2}} d u
$$

The proof of the theorem is given in the next section; it uses an idea of the proof of Theorem 1, [7], vol. I, p. 467.

The theorem stated above is a heavy traffic result. The classical heavy traffic theorem for the GI/G/1 model, cf. [3], Section III.7.2, requires the finiteness of the second moment of $A(t)$ and that of $B(t)$. In a forthcoming paper by O.J. Boxma and the present author, generalizations of the theorem above will be discussed. 


\section{Proof of the Theorem}

We consider first the case with all $c_{n}=0, n=1, \ldots, N$. Consequently, it is seen from (1.1) that we may write: for $t \geq \beta$,

$$
1-B(t)=\frac{c}{(t / \beta)^{\nu}}+F(t)
$$

with

With

$$
\int_{\beta}^{\infty} \mathrm{e}^{-\rho t} F(t) \mathrm{d} t \text { convergent for } \operatorname{Re} \rho>-\delta, \delta>0
$$

we have: for $\operatorname{Re} \rho \geq 0$,

$$
\beta(\rho):=\int_{0-}^{\infty} \mathrm{e}^{-\rho t} \mathrm{~d} B(t), \quad \operatorname{Re} \rho \geq 0
$$

and

$$
\begin{gathered}
\frac{1-\beta(\rho)}{\rho \beta}=\int_{0}^{\infty} \mathrm{e}^{-\rho t}(1-B(t)) \frac{\mathrm{d} t}{\beta} \\
=\int_{0}^{\beta} \mathrm{e}^{-\rho t}\{1-B(t)\} \frac{\mathrm{d} t}{\beta}+\int_{\beta}^{\infty} \mathrm{e}^{-\rho t} \frac{c}{(t / \beta)^{\nu}} \frac{\mathrm{d} t}{\beta}+\int_{\beta}^{\infty} \mathrm{e}^{-\rho t} F(t) \frac{\mathrm{d} t}{\beta},
\end{gathered}
$$

with

$$
1=\int_{0}^{\beta}\{1-B(t)\} \frac{\mathrm{d} t}{\beta}+\int_{\beta}^{\infty} \frac{c}{(t / \beta)^{\nu}} \frac{\mathrm{d} t}{\beta}+\int_{\beta}^{\infty} F(t) \frac{\mathrm{d} t}{\beta} .
$$

It follows that: for $\operatorname{Re} \rho \geq 0$,

$g_{1}(\rho \beta):=\int_{0}^{\beta}\left(1-\mathrm{e}^{-\rho t}\right) \frac{1-B(t)}{\beta} \mathrm{d} t+\int_{\dot{\beta}}^{\infty} \frac{c}{(t / \beta)^{\nu}} \frac{\mathrm{d} t}{\beta}+\int_{\beta}^{\infty}\left\{1-\mathrm{e}^{-\rho t}\right\} F(t) \frac{\mathrm{d} t}{\beta}$.

By using (2.2) it is readily seen that $g_{1}(\rho \beta)$ is a regular function of $\rho$ for $\operatorname{Re} \rho>-\delta$.

For the integral in (2.5) we have by partial integration: for $\operatorname{Re} \rho \geq 0$,

with

$$
1-\frac{1-\beta(\rho)}{\rho \beta}=g_{1}(\rho \beta)-\int_{\beta}^{\infty} \mathrm{e}^{-\rho t} \frac{c}{(t / \beta)^{\nu}} \frac{\mathrm{d} t}{\beta},
$$

$$
c \int_{\beta}^{\infty} \mathrm{e}^{-\rho t}\left(\frac{t}{\beta}\right)^{-\nu} \mathrm{d} \frac{t}{\beta}=-g_{2}(\rho \beta)+c \Gamma(1-\nu)(\rho \beta)^{\nu-1}
$$

$$
g_{2}(\rho \beta):=\frac{c}{\nu-1} \mathrm{e}^{-\rho \beta}+\frac{c \rho}{1-\nu} \int_{0}^{\beta} \mathrm{e}^{-\rho t}\left(\frac{t}{\beta}\right)^{1-\nu} \mathrm{d} t .
$$

Obviously $g_{2}(\rho \beta)$ is an entire function of $\rho$ for all $\rho$; note that $0<\nu-1<1$. 
From cf. [8], p. 3,

$$
\Gamma(\lambda) \Gamma(1-\lambda)=\frac{\pi}{\sin \pi \lambda}, \quad \lambda \text { not an integer }
$$

and with

$$
g(\rho \beta):=g_{1}(\rho \beta)+g_{2}(\rho \beta)
$$

we have from $(2.5), \ldots,(2.10)$ : for $\operatorname{Re} \rho \geq 0$,

$$
1-\frac{1-\beta(\rho)}{\rho \beta}=g(\rho \beta)+\frac{c \pi}{\Gamma(\nu) \sin (\nu-1) \pi}(\rho \beta)^{\nu-1} .
$$

From (2.6), (2.8) and (2.10), it is seen that $g(\rho \beta)$ is also a regular function of $\rho$ for $\operatorname{Re} \rho>-\delta$. From (2.11) it follows that $g(0)=0$. Hence since $g(\rho \beta), \operatorname{Re} \rho>-\delta$ is a regular function we have: for $\operatorname{Re} \rho>-\delta,|\rho| \rightarrow 0$,

$$
g(\rho \beta)=\gamma \rho \beta+\mathrm{O}\left((\rho \beta)^{2}\right)
$$

with $\gamma$ a finite constant.

Write

$$
\alpha(-\rho):=\int_{0}^{\infty} \mathrm{e}^{\rho t} \mathrm{~d} A(t), \quad \operatorname{Re} \rho=0,
$$

so that $\alpha(-\rho)$ is the characteristic function of the distribution $A(t)$. From (1.1), (1.3) and the series expansion of a characteristic function, cf. [10], p. 199, we have: for $\operatorname{Re} \rho=0, \quad|\rho| \rightarrow 0$,

$$
\begin{gathered}
\alpha(-\rho)=1+\alpha \rho+\mathrm{O}\left(|\rho|^{\mu}\right), \\
\alpha:=\int_{0}^{\infty} t \mathrm{~d} A(t)=\beta / a .
\end{gathered}
$$

Let $\mathbf{i}$ be the idle period, i.e., the difference of a busy cycle and the busy period contained in this busy cycle. The relation between the distributions of $\mathbf{w}$ and $\mathbf{i}$ is given by, cf. [4], p. 21, or [3], p. 371: for $\operatorname{Re} \rho=0$,

$$
\mathrm{E}\left\{\mathrm{e}^{-\rho \mathbf{w}}\right\}=\frac{1-\mathrm{E}\left\{\mathrm{e}^{\rho \mathbf{i}}\right\}}{-\rho \mathrm{E}\{\mathbf{i}\}}\left[\frac{1-\beta(\rho) \alpha(-\rho)}{(\beta-\alpha) \rho}\right]^{-1}
$$

note that

$$
\mathrm{E}\{\mathbf{i}\}=(\alpha-\beta) \mathrm{E}\{\mathbf{n}\}
$$

with $\mathbf{n}$ the number of customers served in a busy cycle.

With

$$
A_{\nu}:=\frac{c \pi}{\Gamma(\nu) \sin (\nu-1) \pi}
$$


we have from (2.11): for $\operatorname{Re} \rho=0$,

$$
\begin{aligned}
& \frac{1-\beta(\rho) \alpha(-\rho)}{(\beta-\alpha) \rho} \\
& \quad=\frac{\beta}{\beta-\alpha}\left[\frac{1-\alpha(-\rho)}{\beta \rho}+\{1-g(\rho \beta)\} \alpha(-\rho)-A_{\nu}(\rho \beta)^{\nu-1} \alpha(-\rho)\right] .
\end{aligned}
$$

Set, cf. (1.3),

$$
\sigma:=\min (1, \mu-1)>0 .
$$

By using (2.12) and (2.14) it follows from (2.18) since $\mu>\nu$, cf. (1.3), that: for $\operatorname{Re} \rho=0,|\rho| \rightarrow 0$,

$$
\begin{gathered}
\frac{1-\beta(\rho) \alpha(-\rho)}{(\beta-\alpha) \rho}=\frac{\beta}{\beta-\alpha}\left[-\frac{\alpha}{\beta}+1-A_{\nu}(\rho \beta)^{\nu-1}+\mathrm{O}\left(|\rho|^{\sigma}\right)\right] \\
=1+\frac{a}{1-a}\left\{A_{\nu}(\rho \beta)^{\nu-1}+\mathrm{O}\left(|\rho|^{\sigma}\right)\right\} .
\end{gathered}
$$

Write for $\operatorname{Re} r \geq 0, \mathrm{cf}$. (1.2),

$$
\rho=\left[\frac{1-a}{a} A_{\nu}^{-1}\right]^{\frac{1}{\nu-1}} r / \beta=\Delta r / \beta .
$$

With $\operatorname{Re} \rho \leq 0, a<1$

$$
\omega(\rho):=\mathrm{E}\left\{\mathrm{e}^{-\rho \mathbf{w}}\right\}, \quad \chi(\rho):=\frac{1-\mathrm{E}\left\{\mathrm{e}^{-\rho \mathbf{i}}\right\}}{\rho \mathrm{E}\{\mathbf{i}\}}
$$

we have from (2.15), (2.20) and (2.21): for $0<1-a<<1$ and $\operatorname{Re} r=0$,

(i) $\left.\quad \omega(r \Delta / \beta)=\chi(-r \Delta / \beta)\left[1+r^{\nu-1}+r^{\sigma} \mathrm{O}((1-a))^{\frac{1-\nu+\sigma}{\nu-1}}\right)\right]^{-1}$

(ii) $\omega(r \Delta / \beta)$ and $\chi(r \Delta / \beta)$ are both regular for $\operatorname{Re} r>0$, continuous for $\operatorname{Re} r \geq 0$,

(iii) $|\omega(r \Delta / \beta)| \leq 1, \quad|\chi(r \Delta / \beta)| \leq 1, \operatorname{Re} r \geq 0, \omega(0)=1, \chi(0)=1$.

The conditions (2.23) formulate for $\omega(r \Delta / \beta)$ and $\chi(-r \Delta / \beta)$ a boundary value problem of a type discussed in [6]. It is not difficult to verify that the conditions $(26) i, \ldots, i v$ of $[6]$ are fulfilled for the present boundary value problem with $0<1-a<<1$. Hence from (31) of [6] its solution reads: for $0<1-a<<1$,

$$
\begin{gathered}
\omega(r \Delta / \beta)=\mathrm{e}^{H(r \Delta / \beta)}, \quad \operatorname{Re} r>0, \\
\chi(-r \Delta / \beta)=\mathrm{e}^{H(r \Delta / \beta)}, \quad \operatorname{Re} r<0,
\end{gathered}
$$

with

$$
H(r \Delta / \beta):=\frac{1}{2 \pi \mathrm{i}} \int_{-\mathrm{i} \infty}^{\mathrm{i} \infty} \log \left[1+\eta^{\nu-1}+\eta^{\sigma} \mathrm{O}\left((1-a)^{\frac{1-\nu+\sigma}{\nu-1}}\right)\right] \frac{r \mathrm{~d} \eta}{(\eta-r) \eta}
$$


This integral is a principal value, singular Cauchy integral, cf. [5], Section 1.1.5 and [6]. The integral is absolutely convergent and it follows readily by contour integration in the right half-plane that

$$
\begin{gathered}
\lim _{a \uparrow 1} H(r \Delta / \beta)=\frac{1}{2 \pi \mathrm{i}} \int_{-\mathrm{i} \infty}^{\mathrm{i} \infty} \log \left\{1+\eta^{\nu-1}\right\} \frac{r \mathrm{~d} \eta}{(\eta-r) \eta} \\
=-\log \left\{1+r^{\nu-1}\right\} \quad \text { for } \operatorname{Re} r \geq 0 \\
=0 \quad \text { for } \operatorname{Re} r \leq 0 ;
\end{gathered}
$$

note that the logarithm of the integrand is regular for $\operatorname{Re} \eta>0$, continuous for $\operatorname{Re} \eta \geq 0$ and zero for $\eta=0$, cf. further [5], Section 1.1.5. Hence from (2.24) and $(2.25)$ : for $\operatorname{Re} r \geq 0$,

$$
\begin{gathered}
\lim _{a \uparrow 1} \omega(r \Delta / \beta)=\lim _{a \uparrow 1} \mathrm{E}\left\{\mathrm{e}^{-r \Delta \mathbf{w} / \beta}\right\}=\frac{1}{1+r^{\nu-1}}, \\
\lim _{a \uparrow 1} \chi(r \Delta / \beta)=1 .
\end{gathered}
$$

By using Feller's continuity theorem for L.-S. transforms of probability distributions, it follows that $\Delta \mathbf{w} / \beta$ converges in distribution for $a \uparrow 1$, with limiting distribution $R_{\nu-1}(t)$ given by

$$
\int_{0}^{\infty} \mathrm{e}^{-r t} \mathrm{~d} R_{\nu-1}(t)=\frac{1}{1+r^{\nu-1}}, \quad \operatorname{Re} r \geq 0 .
$$

It remains to prove (1.5). From (2.27) we obtain: for $\operatorname{Re} r \geq 0$,

$$
\int_{0}^{\infty} \mathrm{e}^{-r t}\left\{1-R_{\nu-1}(t)\right\} \mathrm{d} t=\frac{1}{r}\left\{1-\frac{1}{1+r^{\nu-1}}\right\}=\frac{r^{\nu-2}}{1+r^{\nu-1}}
$$

Because $1<\nu<2$, the right-hand side can be continued analytically out from $\operatorname{Re} r \geq 0$, into $\left\{r:|\arg r| \leq \psi, \frac{1}{2} \pi<\psi<\pi\right\}$. With $D$ the contour defined by: for a $r_{0}>0$,

$$
D:=\left\{r: r=r_{0} \mathrm{e}^{\mathrm{i} \phi}, \phi= \pm \psi\right\} \cup\left\{r: r=\operatorname{Re}^{ \pm \mathrm{i} \psi}, R \geq r_{0}\right\}
$$

it is readily shown by starting from the inversion integral for the Laplace transform that

$$
1-R_{\nu-1}(t)=\frac{1}{2 \pi \mathrm{i}} \int_{D} \mathrm{e}^{r t} \frac{r^{\nu-2}}{1+r^{\nu-1}} \mathrm{~d} r
$$

with the direction on $D$ such that on $r=r_{0} \mathrm{e}^{\mathrm{i} \phi}$ it is counterclockwise with respect to the origin. For $r=\left|r_{0}\right|<1$ we have

$$
\frac{r^{\nu-2}}{1+r^{\nu-1}}=\frac{1}{r} \sum_{n=0}^{\infty}(-1)^{n-1} r^{n(\nu-1)} .
$$


We now apply a theorem of Doetsch [7], vol. II, p. 159 to derive an asymptotic series for $1-R_{\nu-1}(t), t \rightarrow \infty$. It is not difficult to show that this theorem may be applied here. It uses the relation

$$
\frac{1}{2 \pi \mathrm{i}} \int_{D} \mathrm{e}^{r t} r^{\lambda} \mathrm{d} \lambda=\frac{1}{\Gamma(-\lambda)} t^{-\lambda-1}, \quad \lambda \neq 0,1,2, \ldots
$$

and it states that: for $t \rightarrow \infty$ and every finite $H \in\{1,2, \ldots\}$,

$$
1-R_{\nu-1}(t)=\sum_{n=1}^{H}(-1)^{n-1} \frac{t^{-n(\nu-1)}}{\Gamma(1-n(\nu-1))}+\mathrm{O}\left(t^{-(H+1)(\nu-1)}\right) .
$$

By using the relation (2.9), the relation (1.5) follows, and the theorem has been proved for the case $c_{n}=0, n=1, \ldots, N$.

To complete the proof for $c_{n}>0$, it suffices to take $c_{1}>0, c_{2}=\ldots=c_{N}=0$, since it is readily seen that the general case proceeds along the same lines. However, we have to distinguish the case that $\nu_{1}(>\nu)$ is not an integer and that of $\nu_{1}$ is an integer $\geq 2$.

First, we consider the case $c_{1}>0, \nu_{1}$ noninteger. Instead of (2.1) we write: for $t \geq \beta$

$$
1-B(t)=\frac{c}{(t / \beta)^{\nu}}+\frac{c_{1}}{(t / \eta)^{\nu_{1}}}+F(t)
$$

with $F(t)$ again satisfying (2.2). By repeated partial integration, it is readily shown, cf. [7], vol. II, p. 468, and (2.7), that: for $\operatorname{Re} \rho \geq 0$,

$$
c_{1} \int_{\beta}^{\infty} \mathrm{e}^{-\rho t} \frac{1}{(t / \beta)^{\nu_{1}}} \frac{\mathrm{d} t}{\beta}=-g_{2}(\rho \beta)+c_{1} \Gamma\left(1-\nu_{1}\right)(\rho \beta)^{\nu_{1}-1},
$$

with $g_{2}(\rho \beta)$ an entire function of $\rho$.

The relation (2.11) is now replaced by: for $\operatorname{Re} \rho \geq 0$,

$$
\begin{gathered}
1-\frac{1-\beta(\rho)}{\beta \rho}=g(\rho \beta)+\frac{c \pi}{\Gamma(\nu) \sin (\nu-1) \pi}(\rho \beta)^{\nu-1} \\
+\frac{c_{1} \pi}{\Gamma\left(\nu_{1}\right) \sin \left(\nu_{1}-1\right) \pi}(\rho \beta)^{\nu_{1}-1}
\end{gathered}
$$

with $g(\rho \beta)$ again a regular function for $\operatorname{Re} \rho>-\delta$ which satisfies $g(0)=0$ and (2.12). Proceeding with the analysis above with (2.11) replaced by (2.35) leads again to $(2.20)$ since $\nu_{1}>\nu$, cf. (1.1). The remaining part of the proof with $c_{1}>0$ does not differ from that with $c_{1}=0$, and so the theorem has been proved for $c_{n}>0$ and $\nu_{n}$ not an integer.

Finally we have to consider the case $\nu_{1}=k \geq 2$, with $k$ an integer. We have, cf. [7], vol. I, p. 468,

$$
c_{1} \int_{\beta}^{\infty} \mathrm{e}^{-s t} \frac{1}{(t / \beta)^{k}} \frac{\mathrm{d} t}{\beta}=-g_{2}(\rho \beta)+c_{1} \frac{(-1)^{k}}{(k-1) !}(\rho \beta)^{k-1} \log (\rho \beta)
$$


again with $g_{2}(\rho \beta)$ a regular function, and the relation (2.11) is now replaced by: for $\operatorname{Re} \rho \geq 0$,

$$
1-\frac{1-\beta(\rho)}{\rho \beta}=g(\rho \beta)+\frac{c \pi(\rho \beta)^{\nu-1}}{\Gamma(\nu) \sin (\nu-1) \pi}+c_{1} \frac{(-1)^{k-1}}{\Gamma(k-1)}(\rho \beta)^{k-1} \log (\rho \beta),
$$

where $g(\rho \beta)$ is again an entire function for $\operatorname{Re} \rho>-\delta$, which satisfies $g(0)=0$ and (2.12). The last term is $\mathrm{o}\left((\rho \beta)^{\nu-1}\right)$ since $k>\nu$. With this it is readily verified that the second equality sign in (2.20) also applies for the present case, and so the remaining part of the proof is similar to that with $c_{1}=0$. Hence the theorem has been proved.

\section{Acknowledgement}

The author thanks Professor O.J. Boxma for several helpful remarks.

\section{References}

[1] Cohen, J.W., On the $M / G / 1$ queue with heavy-tailed service time distributions, Report PNA-R9702, Febr. 1997, CWI, Amsterdam.

[2] Cohen, J.W., The M/G/1 fluid model with heavy-tailed message length distribution, Report PNA-R9714, CWI, Amsterdam.

[3] Cohen, J.W., The Single Server Queue, rev. ed., North Holland, Amsterdam 1982.

[4] Cohen, J.W., On Regenerative Processes in Queueing Theory, Lecture Notes Econ. Math Systems 121, Springer, New York 1976.

[5] Cohen, J.W., Complex functions in queueing theory, Archiv Elektr. Übertragungstechnik 47 (1993), 300-310. (Pollaczek Memorial Volume).

[6] Cohen, J.W. and Boxma, O.J., Boundary Value Problems in Queueing System Analysis, North Holland, Amsterdam 1983.

[7] Doetsch, G., Handbuch der Laplace Transformation, Vol. I, II, III, Birkhäuser, Basel 1950-56.

[8] Erdélyi, A., Higher Transcendental Functions (Bateman Manuscript Project), Vol. 2, McGraw-Hill, New York 1953.

[9] Gnedenko, B.V. and Korolev, V.Y., Random Summation: Limit Theorems and Applications, CRC Press, Boca Raton, Florida 1996.

[10] Loève, M., Probability Theory, 2nd edition, Van Nostrand, Princeton, NJ 1960. 


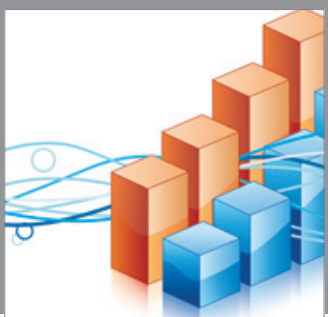

Advances in

Operations Research

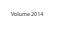

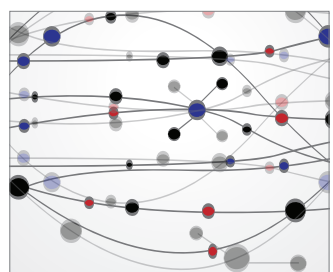

\section{The Scientific} World Journal
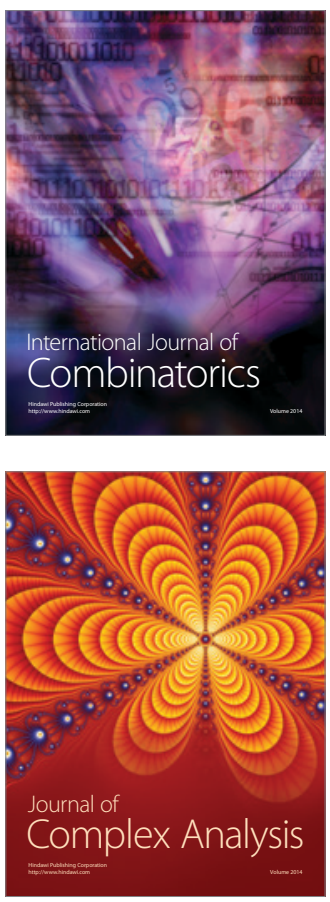

International Journal of

Mathematics and

Mathematical

Sciences
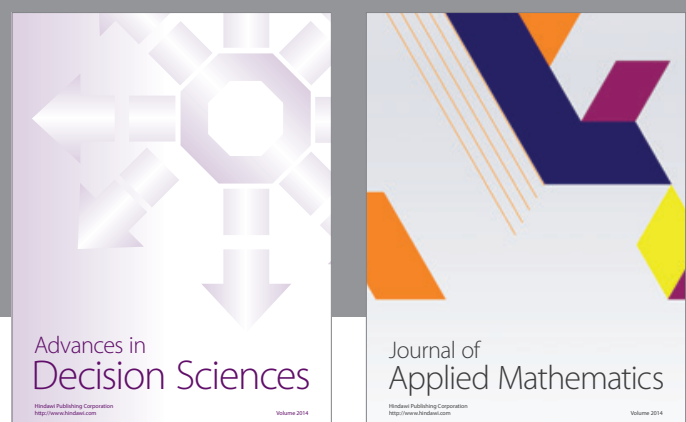

Journal of

Applied Mathematics
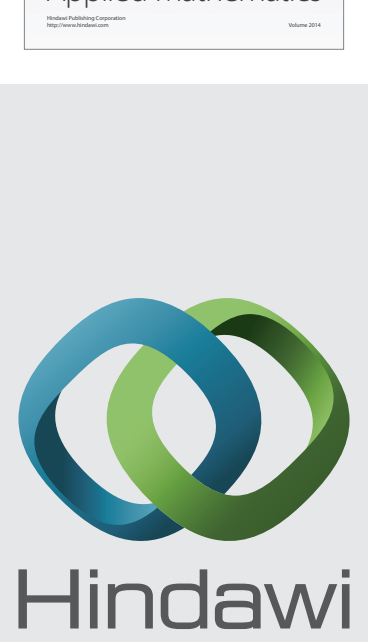

Submit your manuscripts at http://www.hindawi.com
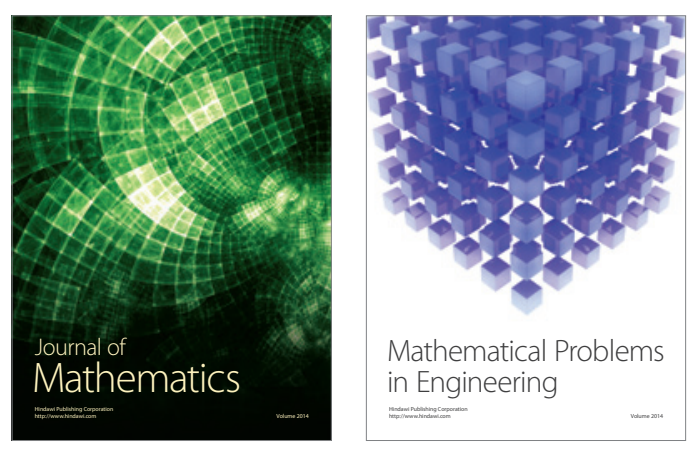

Mathematical Problems in Engineering
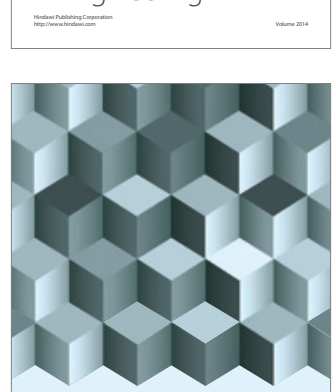

Journal of

Function Spaces
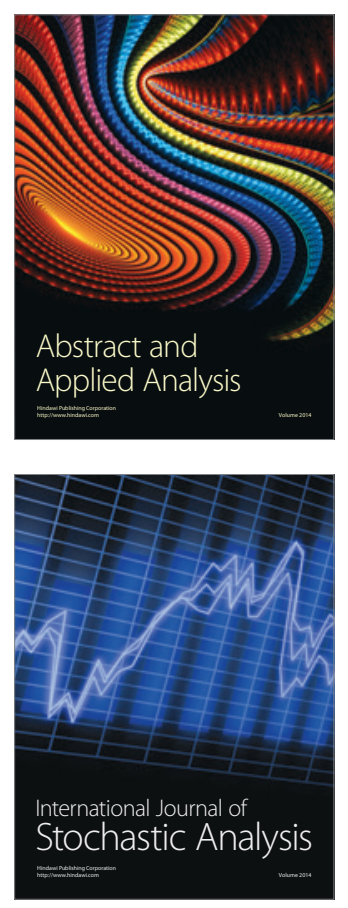

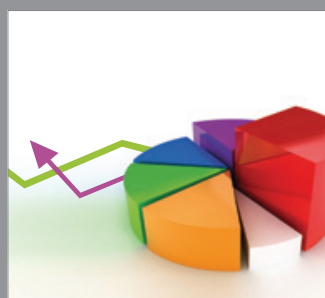

ournal of

Probability and Statistics

Promensencen
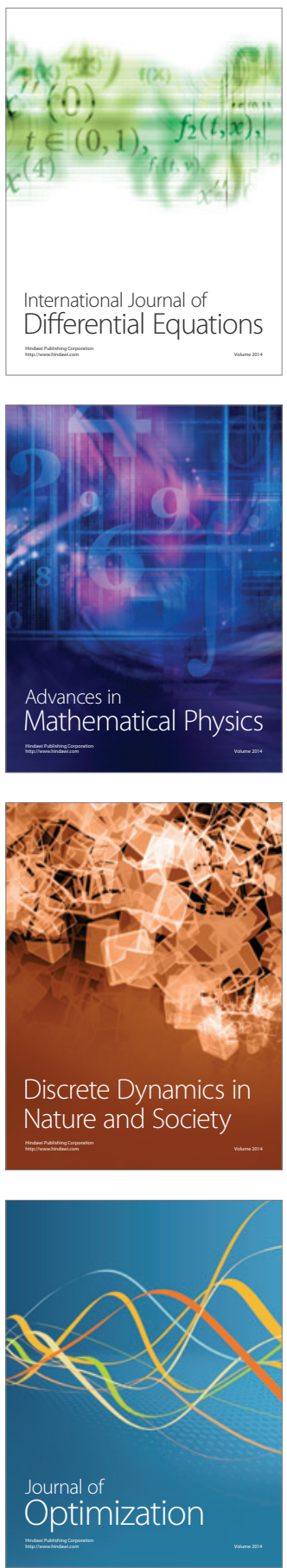